Revue d'histoire du XIXe siècle

Société d'histoire de la révolution de 1848 et des

révolutions du XIXe siècle

$23 \mid 2001$

Nouvelles approches en histoire économique

\title{
Isabelle BACKOUCHE, La trace du fleuve, La Seine et Paris (1750-1850)
}

Paris, Éditions de l'École des hautes études en sciences sociales, 2000.

Florence Bourillon

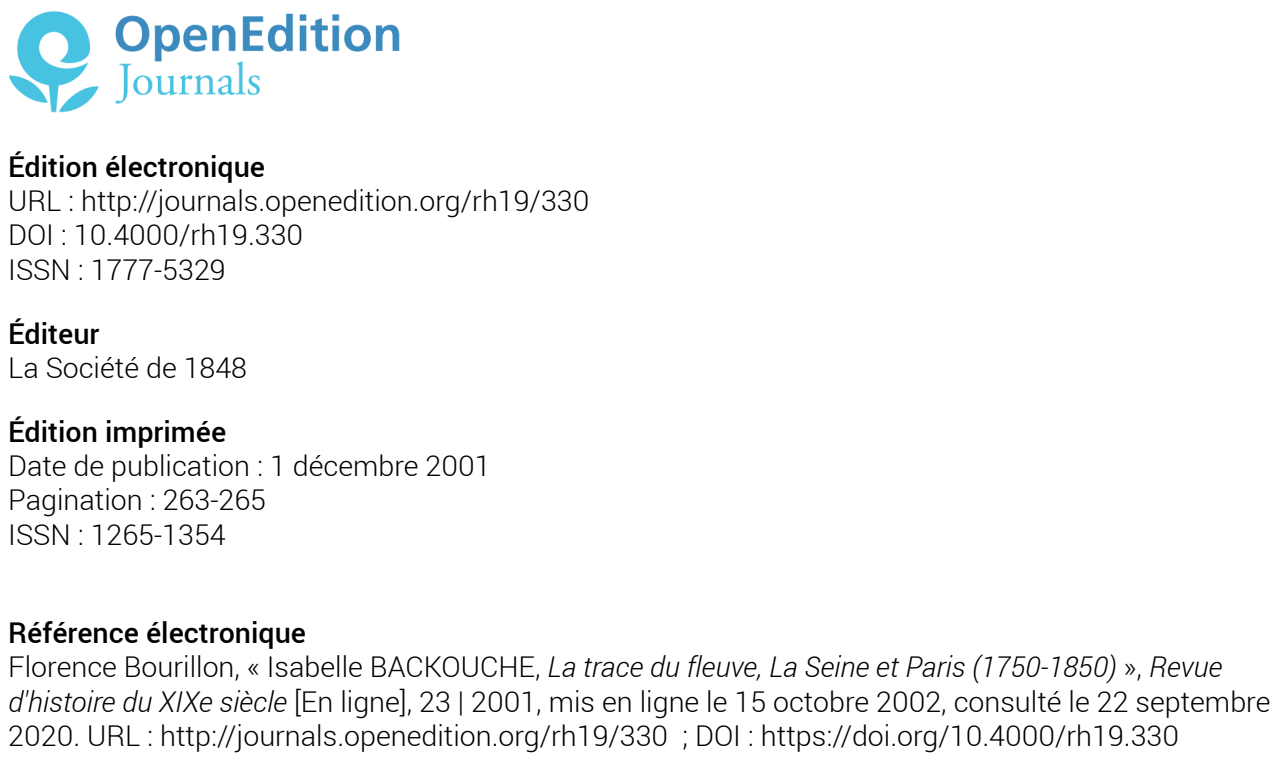

Ce document a été généré automatiquement le 22 septembre 2020.

Tous droits réservés 


\section{Isabelle BACKOUCHE, La trace $d u$ fleuve, La Seine et Paris (1750-1850)}

Paris, Éditions de l'École des hautes études en sciences sociales, 2000.

\section{Florence Bourillon}

Avec La trace du fleuve, la Seine et Paris (1750-1850), Isabelle Backouche livre un ouvrage qui participe au renouvellement de l'historiographie des villes et concoure à l'histoire de la capitale. En soulignant dès la première page que "ce n'est ni l'histoire de Paris, ni l'histoire de la Seine [...] mais celle des relations entre la ville et le fleuve" qui l'intéresse, l'auteur met en lumière l'intérêt de sa démarche. L'étude évite de se cantonner au récit d'une histoire du fleuve --et de son délaissement-- et tient compte des caractères particuliers de la ville. Est ainsi énoncé l'objet de la recherche, un espace urbain "original", la Seine, espace construit qui concilie deux approches : celle du fleuve dans la ville et celle des Parisiens sur le fleuve, à la jonction d'une histoire des formes, des usages et des représentations. Isabelle Backouche identifie de la sorte un "terrain d'expérimentation fructueux" ouvert à un questionnement large qui porte aussi bien sur les acteurs, les activités, les pratiques, les projets que sur le commerce de Paris et de sa région ou les relations centre-périphérie. L'espace fluvial se dilate ou se réduit au gré des questions posées. Le choix des diverses échelles d'observation permet de rendre compte de la dynamique des pratiques qu'il abrite ou des décisions ou projets qui le concernent, et renvoie aux perceptions que les contemporains en ont. L'occasion de l'observation est fournie par les situations de conflits entre les différents intervenants, consommateurs, commerçants, riverains, autorités, celle de la ville représentée par le prévôt des marchands ou celle du roi par le lieutenant général de police puis, plus tard celle des préfets.

À l'origine de la recherche, un constat : la Seine est aujourd'hui étrangère à la capitale ; les quais servent à la circulation automobile et le fleuve est réservé à la navigation. Quand et comment la Seine a-t-elle perdu le rôle "identitaire" qu'elle occupait ? C'est une histoire de la rupture entre la ville et son fleuve qui est ici rapportée, selon une périodisation qui échappe aux repères classiques ou attribuerait au seul XIX ${ }^{\mathrm{e}}$ siècle la conception des grands projets de rénovation. 
La Seine est au milieu du XVIII e siècle un "espace partagé" au cœur de la ville : fleuve nourricier --près des deux tiers des consommations parisiennes transitent par les ports et la moitié d'une eau "rare et précieuse" y est puisée-- voie de brassage de population par les coches d'eau qui relient les villes de la haute et de la basse Seine, site des joutes ou des démonstrations festives offertes par la Monarchie, la Seine est aussi le lieu de vie d'une population nombreuse : porteurs d'eau, blanchisseuses, meuniers, portefaix, pêcheurs, cuiseurs de tripes..., promeneurs ou commerçants attirés par la présence de la foule. Le fleuve détermine ainsi un monde urbain singulier associant à ses rives, ses quais, ses ports, ses grèves ou ses ponts recouverts de maisons, la ville entière. Pourtant l'espace fluvial apparaît discontinu : à une opposition entre l'amont et l'aval due à une spécialisation des ports et des activités qui en dépendent, s'ajoute une fragmentation du cours du fleuve en bassins délimités par les ponts. Ainsi, profondément intégré dans la ville, le fleuve est devenu facteur des différenciations urbaines qui se renforcent au cours du siècle. Dans le même temps, la concurrence des usages s'exacerbe et la régulation tentée par des pouvoirs rivaux accentue les divergences : les conflits révèlent une hiérarchisation des pratiques qui se fait en faveur de la circulation. La seconde partie de l'ouvrage est consacré à la remise en cause de l'espace urbain tel qu'il apparaissait dans les années 1750 . Les projets très nombreux répondent à des exigences nouvelles à la fois économiques, esthétiques et de santé publique, négligeant la diversité des usages et des pratiques riveraines du fleuve. Entre 1758 et 1769, le prévôt des marchands et le roi prévoient un programme général d'aménagement. Le fleuve devient "l'épine dorsale d'un projet à l'échelle de la capitale" : il s'agit d'améliorer la navigation de l'Ile Louviers à la barrière des Invalides, d'assurer une circulation continue le long du fleuve grâce à l'aménagement des quais réhaussés, de détruire les maisons sur les ponts, d'en construire de nouveaux. Les travaux retardés par des difficultés financières et la résistance des riverains, commencent en 1786 et transforment en trois ans, l'aspect du fleuve.

Les projets et les premières réalisations vont modifier le regard sur la Seine. C'est à la fois la banalisation du fleuve et la recherche de la cohérence des aménagements et des pratiques qui vont présider aux projets d'ouverture à l'espace national. Du projet urbain on passe au projet fluvial, prévoyant l'aménagement de gares, de zones d'entrepôt. Les premières années du XIX ${ }^{\mathrm{e}}$ siècle en confiant aux préfets et aux ingénieurs des Ponts et Chaussées la réalisation des aménagements, confirment le rôle de la Seine devenue une section de la grande voie de navigation conçue dans une logique de développement qui se fait à l'échelle nationale. Le fleuve devient ainsi "étranger à sa ville".

Au total, un ouvrage d'une très grande richesse qui participe à la réflexion et aux interrogations sur les sources, les méthodes et l'écriture de l'histoire urbaine. On soulignera l'intérêt d'une démarche qui définit les caractères spécifiques de la ville ("l'urbanité ?") à travers la construction d'un espace transversal, la nouveauté des sources et l'attention croisée portée, parmi d'autres, aux pratiques urbaines, aux projets d'aménagement ou aux décisions du pouvoir. Ajoutons que les travaux $\mathrm{d}^{\prime}$ Isabelle Backouche ouvrent de nombreuses perspectives aux chercheurs du XIX ${ }^{\mathrm{e}}$ siècle parisien. Le décloisonnement chronologique situe la trace du fleuve dans une histoire aux temporalités inégales, marquée par la lente disparition des usages riverains ou les interventions plus radicales des travaux d'aménagement : les plaintes des élus de la rive gauche sous la monarchie de Juillet, la crainte du "déplacement de Paris" vers le nord-ouest, les projets de recentrage sur la Cité, la destruction des péages 
sur les ponts aux premiers jours de février 1848, la population sur les quais, laissent entrevoir la présence du fleuve et permet de comprendre autrement les grands travaux de la seconde moitié du siècle. 